\title{
Incidencia natural de parasitoides de huevos de Oebalus insularis Stal (Heteroptera: Pentatomidae) en Panamá
}

\author{
Natural incidence of eggs parasitoids of Oebalus insularis Stal \\ (Heteroptera: Pentatomidae) in Panama
}

\author{
Bruno Zachrisson $^{1 *}$, Valmir Costa ${ }^{2}$, Juan Bernal ${ }^{3}$
}

\section{RESUMEN}

El objetivo del trabajo fue el de determinar la tasa de parasitismo en huevos de Oebalus insularis en diferentes variedades (CONAGRO-2, ESTRELLA-92, IDIAP-5405) de arroz. La tasa de parasitismo de los huevos de O. insularis se determinó en condiciones controladas de temperatura $\left(25 \pm 1^{\circ} \mathrm{C}\right)$, humedad relativa $(85 \pm 3 \%)$ y fotofase (12 horas). Se identificaron los parasitoides Telenomus podisi y Trissolcus basalis. La tasa de parasitismo promedio del complejo de parasitoides fue superior al $90 \%$ y la especie más abundante fue T. podisi, con $81,8 \%$ de parasitismo.

Palabras clave: Control biológico, Oebalus insularis, Telenomus podisi, Trissolcus basalis, Oryza sativa.

\begin{abstract}
The objective of this work was to determine the parasitism rate of Oebalus insularis eggs, in different rice varieties (CONAGRO-2, ESTRELLA-92, IDIAP-5405). The parasitism rate of $\mathrm{O}$. insularis eggs was determinated under controlled conditions of temperature $\left(25 \pm 1{ }^{\circ} \mathrm{C}\right)$, relative humidity $(85 \pm 3 \%)$ and photophase $(12$ hours). The parasitoids Telenomus podisi and Trissolcus basalis were identified. The average parasitismo rate of parasitoids complex was above $90 \%$ and the most abundant specie was Te. podisi, with $81,8 \%$ parasitism.
\end{abstract}

Key words: Biological control, Oebalus insularis, Telenomus podisi, Trissolcus basalis, Oryza sativa.

El arroz (Oryza sativa L.) es uno de los cultivos más importantes a nivel mundial, el que está presente en más de 113 países y contribuye a la alimentación de la mitad de la población del mundo (FAO, 2006). Este rubro aporta el 50\% del total de calorías a la dieta de la población panameña (Puga, 2009). La reducción en el rendimiento de este cultivo, provocado por la incidencia de insectos-plagas, corresponde aproximadamente a 12\% (Vivas y Notz, 2009; 2011). El "chinche del arroz", como es conocido Oebalus insularis Stal, 1872 (Heteroptera: Pentatomidae), es una de las plagas más importantes en el cultivo del arroz para Panamá y América Central (Cherry y Nuessly, 2010; Rodríguez et al., 2006). Las ninfas y adultos de $O$. insularis recién eclosionadas inoculan toxinas y fitopatógenos al succionar el contenido de los granos en estado lechoso (Zachrisson y Martínez, 2011). Este síntoma conocido como "manchado de grano" guarda relación con las infestaciones de $O$. insularis que a partir de índices superiores a 0,7 insectos/panículas afecta cualitativa y cuantitativamente el rendimiento del cultivo (Ferreira y Barrigossi, 2006; Rodríguez et al., 2006; Vivas y Notz, 2009; 2010). Por lo que el parasitismo natural en la fase de huevo de la plaga es la opción más viable y eficiente para reducir la población del insecto, garantizando la sostenibilidad de este rubro agrícola (Rodríguez et al., 2006; Zachrisson y Martínez, 2011). En América Central se han registrado varias especies de parasitoides oófagos de $O$. insularis, reportándose

\footnotetext{
1 Instituto de Investigación Agropecuaria de Panamá, Ciudad del Saber, edificio 611-612, Apartado Postal 832-2758, Panamá, Panamá.

2 Instituto Biológico, Rodovia Heitor Penteado, km 3, Caixa Postal 70, CEP- 13001-970 Campinas, SP, Brasil.

3 Escuela de Biología, Facultad de Ciencias Naturales y Exactas. Universidad Autónoma de Chiriquí, Apartado Postal 0427, David, Panamá.

* Autor para correspondencia: bruno.zachrissons@idiap.gob.pa
} 
Telenomus latrifrons Ashmead, 1896 y Telenomus podisi (Ashmead, 1881) (Scelionidae: Telenominae) (Shannon, 1989). Zachrisson y Martínez (2011) confirmaron el elevado potencial de T. podisi como agente de control de $O$. insularis, reportando por primera vez el parasitismo natural de T. podisi y de T. basalis en Panamá. No obstante, la interacción del "parasitoide-huésped-planta" es un proceso dinámico relevante para la implementación de programas de control biológico dirigido al manejo de O. insularis (Zachrisson y Martínez, 2011). Margaria et al. (2009) destaca la necesidad de conocer la asociación "parasitoide-huésped", en donde compuestos volátiles como las kairomonas pueden influir en esta interacción (Moraes et al., 2008). Sin embargo, el incremento de la temperatura registrada en la última década, para las principales zonas arroceras de Panamá (Puga, 2009), sugiere la necesidad de realizar ajustes periódicos en programas de control biológico (Hance et al., 2007). Estudios que determinen el parasitismo natural de huevos de $O$. insularis proporcionan subsidios para la selección de parasitoides potenciales de control, que presenten características biológicas y reproductivas ideales, adaptadas a las condiciones agroclimáticas del cultivo (Bueno et al., 2009). Por consiguiente, este trabajo tuvo como objetivo determinar la tasa de parasitismo en huevos de $O$. insularis en variedades de arroz liberadas recientemente en Panamá. La recolección de huevos de $O$. insularis se realizó en parcelas experimentales, libre de la aplicación de insecticidas, entre las fases fenológicas de floración y grano lechoso. Durante los meses de junio y diciembre del año agrícola
2012 se muestrearon las parcelas experimentales ubicadas en Tocumen, provincia de Panamá, Panamá (09 03'93"N; 79²0'13"O); Chichebre, provincia de Panamá, Panamá (0907'67'N; 79¹3'78”O); Paso Blanco, provincia de Panamá $\left(09^{\circ} 05^{\prime} 52^{\prime \prime} \mathrm{N}\right.$ $79^{\circ} 10^{\prime} 63^{\prime}$ 'W) y Juan Hombrón, provincia de Coclé, Panamá ( $08^{\circ} 23^{\prime} 83^{\prime}$ 'N ; $08^{\circ} 13^{\prime} 90^{\prime}$ 'O). Las variedades evaluadas por localidad fueron: a) CONAGRO-2 (Tocumen, provincia de Panamá), b) ESTRELLA-92 (Paso Blanco; provincia de Panamá), c) IDIAP5405 (Chichebre, provincia de Panamá; Juan Hombrón, provincia de Coclé). La temperatura, precipitación pluviométrica y humedad relativa presentaron intervalos de variación reducida en las localidades evaluadas, con promedios de $30,1^{\circ} \mathrm{C}$, $235,4 \mathrm{~mm}$ y $86,7 \%$, respectivamente. Los muestreos realizados en parcelas de 2 ha consideraron 20 plantas en diez puntos del área experimental, seleccionadas aleatoriamente. Las masas de huevos de $O$. insularis recolectadas fueron transferidas al laboratorio e individualizadas en platos Petri de vidrio $(9,0 \times 1,5 \mathrm{~cm})$, manteniendo la humedad interna por medio de papel filtro humedecido con agua destilada, que era colocada en la base del plato. Posteriormente el material fue transferido a cámaras climatizadas, reguladas a $25 \pm 1{ }^{\circ} \mathrm{C}$ de temperatura, $85 \pm 3 \%$ de humedad relativa y fotofase de 12 horas. Los parasitoides emergidos fueron fijados en viales de $1 \mathrm{ml}$ conteniendo etanol al $70 \%$ e identificados según Johnson (1984; 1985). Las especies identificadas se depositaron en la colección de insectos entomófagos "Oscar Monte”, del Instituto Biológico Campinas, Brasil. El porcentaje de parasitismo se determinó por medio

Tabla 1. Porcentaje de huevos de Oebalus insularis parasitados recolectados en diferentes variedades de arroz en cuatro localidades productoras de arroz de Panamá.

\begin{tabular}{|c|c|c|c|c|c|c|}
\hline Localidad & Variedad & $\begin{array}{l}\text { Número } \\
\text { de huevos } \\
\text { colectados }\end{array}$ & $\begin{array}{l}\text { Número } \\
\text { de huevos } \\
\text { parasitados }\end{array}$ & $\begin{array}{c}\text { Porcentaje de } \\
\text { parasitismo }(\%)\end{array}$ & $\begin{array}{c}\text { Porcentaje de } \\
\text { parasitismo (\%) } \\
\text { Telenomus podisi }\end{array}$ & $\begin{array}{c}\text { Porcentaje de } \\
\text { parasitismo } \\
\text { (\%) Trissolcus } \\
\text { basalis }\end{array}$ \\
\hline $\begin{array}{l}\text { Tocumen } \\
\text { (Provincia de Panamá) }\end{array}$ & CONAGRO-2 & 510 & 480 & 94,1 & $85,7(437)^{1}$ & $8,4(43)^{1}$ \\
\hline $\begin{array}{l}\text { Paso Blanco } \\
\text { (Provincia de Panamá) }\end{array}$ & Estrella-92 & 425 & 394 & 92,7 & 81,4 (346) & $11,3(48)$ \\
\hline $\begin{array}{l}\text { Chichebre } \\
\text { (Provincia de Panamá) }\end{array}$ & IDIAP-5405 & 561 & 501 & 89,3 & 80,0 (449) & $9,3(52)$ \\
\hline $\begin{array}{l}\text { Juan Hombrón } \\
\text { (Provincia de Coclé) }\end{array}$ & IDIAP-5405 & 665 & 593 & 89,2 & 80,3 (534) & $8,9(59)$ \\
\hline
\end{tabular}

(1) Número de parasitoides emergidos de los huevos de O. insularis. 
de la fórmula $\mathrm{P}(\%=$ número de huevos parasitados/ número total de huevos sometidos al parasitismo) х100. T. podisi y $T$. basalis fueron las especies recolectadas en las áreas experimentales evaluadas (Tabla 1), confirmándose la asociación existente entre $O$. insularis y el complejo de parasitoides de huevos en Panamá. La especie que presentó mayor abundancia fue $T$. podisi, registrando parasitismo entre 80 y $85 \%$, seguida por $T$. basalis (Tabla 1). La eficiente asociación registrada entre $T$. podisi у $O$. insularis es sustentada por la elevada tasa de parasitismo, encontrada en las diferentes áreas de producción de arroz. Por lo que se recomienda T. podisi como un agente promisorio de control de O. insularis para posteriores estudios biológicos y reproductivos de este parasitoide, en condiciones abióticas controladas. Estos parámetros servirán de base para programas de multiplicación masiva de $T$. podisi, que facilite su liberación, en las áreas de producción.

\section{Literatura Citada}

Bueno, R.C.O.F.; Parra, J.R.P.; Bueno, A.P.; Haddad, M.L. 2009. Desempenho de trichogrammatídeos como potenciais agentes de controle de Pseuplosia includens Walker (Lepidoptera: Noctuidae). Neotropical Entomology, 38 (3): 389-394.

Cherry, R.; Nuesly, G.

2010. Establishment of a new stink bug pest, Oebalus insularis (Hemiptera: Pentatomidae), in Florida rice. Florida Entomologist, 93: 291-293.

FAO

2006. Seguimiento del mercado del arroz, Roma http://www. ftp.fao.org/docrep/fao/009/ag068s00.pdf. Consultado: 1/8/12.

Ferreira, E.; Barrigossi, J.A.F.

2006. Produção e qualidade do grão irrigado infestado por adultos de percevejo-das-panículas. Pesquisa Agropecuária Brasileira, 41: 1083-1091.

Hance, T.; Van Baaren, J.; Vernon. P.; Boivin, G.

2007. Impact extreme temperatures on parasitoids in a climate change perspective. Annual Review of Entomology, 107-126.

Johnson, N.F.

1984. Systematics of Neartic Telenomus. Classification and revisions of podisi and phymate species groups (Hymenoptera: Scelionidae). Bulletin of the Ohio Biological Survey, 6: 1-113. Johnson, N.F.

1985. Systematics of New World Trissolcus (Hymenoptera: Scelionidae): species related to T. basalis. Canadian Entomologists, 117: 431-445.

Margaría, C.B.; Loiácono, M.S.; Lanteri, A.A.

2009. New geographic and host records for scelionid wasps (Hymenoptera: Scelionidae) parasitoids of insects pests in South America. Zootaxa, 2314: 41-49.

Moraes, M.C.B.; Pareja, M.F.; Laumann, R.A.; HoffmannCampo, C.B.; Borges, C.B.

2008. Response of the parasitoid Telenomus podisi to induced volatiles from soybean damaged by stink bug herbivory and ovipostion. Journal of Plant Interactions, 3 (2): 1742-1756.
Puga, B.E.S.

2009. Informe final del año agrícola (2008-2009). Panamá: Ministerio de Desarrollo Agropecuario, Programa nacional de granos básicos en el cultivo de arroz mecanizado, secano y riego, pp. 1-31.

Rodriguez, P.G.; Navas, D.; Medianero, E.; Chang, R.

2006. Cuantificación del daño ocasionado por Oebalus insularis (Heteroptera: Pentatomidae) en el cultivo del arroz (Oryzica-1) en Panamá. Revista Colombiana de Entomología, 32: 131-135.

Shannon, P.

1989. Programas de MIP Implementados: Arroz. En: Manejo Integrado de Plagas Insectiles en la Agricultura: Estado Actual y Futuro. 1ra Edición, Andrews, K. y Quesada, J. R. 1989. Editora AID. Escuela Agrícola Panamericana, Zamorano, Honduras, pp. 567-586.

Vivas, L.E.; Notz, A.

2009. Plan de muestreo secuencial de Oebalus insularis Stal (Hemiptera: Pentatomidae), en el cultivo de arroz en Calabozo estado de Guárico, Venezuela. Revista Científica UDO Agrícola, 9: 857-892.

Vivas, L.E.; Notz, A.

2010. Determinación del umbral y nivel de daño económico del chinche vaneador del arroz, sobre la variedad cimarrón en Calabozo estado de Guárico, Venezuela. Agronomía Tropical, 60: 271-281.

Vivas, L.E.; Notz, A.

2011. Distribución espacial en poblaciones de Oebalus insularis Stal (Hemiptera: Pentatomidae) en el cultivo de arroz, estado Guárico, Venezuela. Revista Científica UDO Agrícola, 11: 109-125.

Zachrisson, B.A.; Martínez, O.

2011. Bioecologia de Telenomus podisi (Ashmead) y Trissolcus basalis (Wollaston) (Himenoptera: Scelionidae), parasitoides oófagos de Oebalus insularis (Kulghast) (Heteroptera: Pentatomidae). Tecnociencia, 13: 65-76. 
